\title{
Waveguide bands for a system of macromolecules
}

\author{
V. M. Adamyan ${ }^{1}$, I. V. Blinova ${ }^{2}$, A. I. Popov², I. Yu. Popov² \\ ${ }^{1}$ Odessa I. I. Mechnikov National University, Dvoryanskaya str., 2, Odessa, 65082, Ukraine \\ 2 ITMO University, Kronverkskiy, 49, Saint Petersburg, 197101, Russia \\ popov1955@gmail.com
}

PACS 72.80.Le; 03.65 Nk

DOI 10.17586/2220-8054-2015-6-5-611-617

\begin{abstract}
A system of parallel chain-type macromolecules (linear polymers) is considered. The spectrum of an electron in such a system is described. Waveguide bands are shown to be present, ensuring conductivity. Consideration is undertaken within the framework of a zero-range potentials model based on the theory of self-adjoint extensions of symmetric operators. Possible applications also discussed.
\end{abstract}

Keywords: spectrum, linear polymer, zero-range potential.

Received: 15 September 2015

Revised: 25 September 2015

\section{Introduction}

This paper deals with the theoretical investigation of a nanosystem which can be used for the creation of optically controlled magnetoresistance. The possibilities for this type of control were outlined in [1]. Layered structures having magnetic layers and a spacer filled with conducting polymer (linear molecules) with inserted banana-shaped photosensitive molecules were suggested to be used for this purpose. Linear polymeric molecules should ensure electronic conductivity between the magnetic layers. In the present paper, we investigate the electronic band structure for a system of linear molecules.

Consider an infinite periodic chain of impurity atoms placed along some line (let it be the axis $O X$ ) between two nanolayers (magnetic). We use the effective mass approximation, i.e. we take into account the influence of the nanolayers by changing the effective mass of the electron in the spacer (see, e.g., [2]). In chosen coordinate system, the position of $n$-th atom of the chain $(n=0, \pm 1, \pm 2, \ldots)$ is given by a vector $n \mathbf{a}=(n a, 0,0)$, where $a$ is the chain period. A simple but less accurate quantum graph model of such systems (see, e.g., [3,4]) is used more often. To compute the energy spectrum of the chain, we use the zero-range potentials model. We mention that the spectrum for chain structures in $\mathbb{R}^{3}$ can be rather unusual (see, e.g., [5]), but in our periodic case it has a defined band structure. As for zero-range potentials, the background for the model is formed by the theory of self-adjoint extensions of symmetric operators (see, e.g., $[6,7]$ ). Namely, one starts from a self-adjoint operator - the Laplacian in $L_{2}\left(\mathbb{R}^{3}\right)$ with the domain $W_{2}^{2}\left(\mathbb{R}^{3}\right)$. Here $W_{2}^{2}$ is the Sobolev space. Let us restrict the operator to the set of smooth functions which vanish at points $p_{n}=|\mathbf{r}-\mathbf{n a}| ; \quad n=0, \pm 1, \ldots$. The closure of this restricted operator is a symmetric operator with infinite deficiency indices. To construct a self-adjoint extension, it is more convenient to deal with the corresponding restriction of the adjoint operator. There are several ways to describe extensions, e.g., boundary triplets method ( [8,9], von Neumann formulas ( $[10])$, Krein resolvent formula $([11,12])$. We will use here a variant of the second 
approach which allows one, in the case of semi-boundedness of the Hamiltonian, to present an element from the domain of the adjoint operator in the following form:

$$
\psi(\mathbf{r})=\psi_{0}(x)+\sum_{m=-\infty}^{\infty} C_{m} \cdot \frac{e^{-i k_{0}|\mathbf{r}-m \mathbf{a}|}}{4 \pi|\mathbf{r}-m \mathbf{a}|},
$$

where $\psi_{0}$ belongs to the domain of the Friedrichs extension of the initial symmetric operator, $C_{m}$ is some constant, $k_{0}=\sqrt{\lambda_{0}}, \lambda_{0}$ is a regular value of the spectral parameter (particularly, one can choose real negative value of $\left.\lambda_{0}, \Im k_{0}>0\right)$. To explain the choice of extension, it is convenient to consider the simplest case of a single point-like potential (at $\mathbf{r}=0$ ). In this case, one has the symmetric operator with the deficiency indices $(1,1)$. The domain of the adjoint operator $\left(A^{*}\right)$ consists of the following elements (compare with (1)):

$$
\psi(\mathbf{r})=\psi_{0}(x)+C_{0} \frac{e^{-i k_{0}|\mathbf{r}|}}{4 \pi|\mathbf{r}|} .
$$

To construct a self-adjoint extension, one calculates the boundary form for elements $\psi, \phi$ from the domain of the adjoint operator:

$$
\left(A^{*} \psi, \phi\right)-\left(\psi, A^{*} \phi\right)=\psi_{0}(0) \overline{C_{0}^{\phi}}-\overline{\phi_{0}(0)} C_{0}^{\psi} .
$$

Evidently, one gets a self-adjoint extension (annihilation of this form) if there is the following relation between $\psi_{0}(0)$ and $C_{0}^{\phi}$ :

$$
\psi_{0}(0)=b \quad C_{0}^{\psi}, \Im b=0 .
$$

Formally, the condition (2) takes the form of a "boundary condition" at the point:

$$
\lim _{|\mathbf{r}| \rightarrow 0}\left[\frac{\partial}{\partial|\mathbf{r}|}-b\right]|\mathbf{r}| \Psi=0 .
$$

One often rewrites condition (3) as a condition for the logarithmic derivative:

$$
\lim _{|\mathbf{r}| \rightarrow 0} \frac{1}{|\mathbf{r}| \Psi} \frac{\partial}{\partial|\mathbf{r}|}(|\mathbf{r}| \Psi)=b
$$

For the case of the periodic chain, one has:

$$
\begin{gathered}
\lim _{p_{n} \rightarrow 0}\left[\frac{\partial}{\partial p_{n}}-b\right] p_{n} \Psi=0, \\
p_{n}=|\mathbf{r}-\mathbf{n a}| ; \quad n=0, \pm 1, \ldots .
\end{gathered}
$$

Below we will use this form of the condition.

\section{Two chains}

Consider the spectral problem for two parallel chains of zero-range potentials in $\mathbb{R}^{3}$. We assume that centers of the chains are shifted by vector $\mathbf{g}=\left(g_{1}, g_{2}, 0\right)$. The first chain has centers at points $n \mathbf{a}=n a \mathbf{e}_{\mathbf{1}}$, the second chain - at points $n \mathbf{a}+\mathbf{g}=\left(n a+g_{1}\right) \mathbf{e}_{\mathbf{1}}+g_{2} \mathbf{e}_{\mathbf{2}}$; $n=0, \pm 1, \pm 2, \ldots ; \mathbf{e}_{\mathbf{1}}, \mathbf{e}_{\mathbf{2}}$ are unit vectors of axis $X$ and axis $Y$, correspondingly. The Green function for the operator with point-like potentials has the form:

$$
\begin{gathered}
G\left(\mathbf{r}, \mathbf{r}^{\prime}, E\right)=\frac{1}{4 \pi} \frac{\exp \left[i s\left|\mathbf{r}-\mathbf{r}^{\prime}\right|\right]}{\left|\mathbf{r}-\mathbf{r}^{\prime}\right|}+ \\
\sum_{n=-\infty}^{+\infty}\left\{C_{n}^{(1)} \frac{\exp [i s|\mathbf{r}-n \mathbf{a}|]}{|\mathbf{r}-n \mathbf{a}|}+C_{n}^{(2)} \frac{\exp [i s|\mathbf{r}-n \mathbf{a}-\mathbf{g}|]}{|\mathbf{r}-n \mathbf{a}-\mathbf{g}|}\right\} .
\end{gathered}
$$


Coefficients $C_{n}^{(1)}, C_{n}^{(2)}$ are determined for the system given by conditions (5), which gives one the following system after the Fourier transform:

$$
\begin{aligned}
& D(k, s) \xi_{k}^{(1)}+Q(k, s) \xi_{k}^{(2)}=\varphi_{k}\left(\mathbf{r}^{\prime}\right) \\
& \overline{Q(k, s)} \xi_{k}^{(1)}+D(k, s) \xi_{k}^{(2)}=\varphi_{k}\left(\mathbf{r}^{\prime}\right)
\end{aligned}
$$

where

$$
\begin{gathered}
\xi_{k}^{(j)}=\sum_{n} C_{n}^{(j)} e^{i k n a} ; \quad j=1,2, \\
\varphi_{k}(\mathbf{r})=-\frac{1}{4 \pi} \sum_{n} \frac{\exp [i s|\mathbf{r}-n \mathbf{a}|+i k n a]}{|\mathbf{r}-n \mathbf{a}|} \\
D(k, s)=\sum_{n \neq 0} \frac{\exp [i s|n| a+i k n a]}{|n| a}+i s-b \\
Q(k, s)=\sum_{n} \frac{\exp [i s|n \vec{a}-\mathbf{g}|+i k n a]}{|n \mathbf{a}-\mathbf{g}|}
\end{gathered}
$$

Hence,

where

$$
\begin{aligned}
& \xi_{k}^{(1)}=\frac{1}{2}\left\{\begin{array}{l}
\frac{\varphi_{k}\left(\mathbf{r}^{\prime}\right)+\chi \varphi_{k}\left(\mathbf{r}^{\prime}-\mathbf{g}\right)}{D(k, s)+|Q(k, s)|}+\frac{\varphi_{k}\left(\mathbf{r}^{\prime}\right)-\chi \varphi_{k}\left(\mathbf{r}^{\prime}-\mathbf{g}\right)}{D(k, s)-|Q(k, s)|} \\
\xi_{k}^{(2)}=\frac{\bar{\chi}}{2}\left\{\frac{\varphi_{k}\left(\mathbf{r}^{\prime}\right)+\chi \varphi_{k}\left(\mathbf{r}^{\prime}-\mathbf{g}\right)}{D(k, s)+|Q(k, s)|}-\frac{\varphi_{k}\left(\mathbf{r}^{\prime}\right)-\chi \varphi_{k}\left(\mathbf{r}^{\prime}-\mathbf{g}\right)}{D(k, s)-|Q(k, s)|}\right.
\end{array}\right\}
\end{aligned}
$$

$$
\chi=\chi(k, s)=\frac{Q(k, s)}{|Q(k, s)|}
$$

Returning from $\xi_{k}^{(i)}$ to coefficients $C_{n}^{(i)}$ using formulas:

$$
C_{n}^{(i)}=\frac{a}{2 \pi} \int_{-\pi / a}^{+\pi / a} \xi_{k}^{(i)} e^{-i k n a} d k
$$

one obtains the following expression for the Green function:

$$
\begin{gathered}
G\left(\mathbf{r}, \mathbf{r}^{\prime}, E\right)=G_{0}\left(\mathbf{r}-\mathbf{r}^{\prime}, E\right)-a \int_{-\pi / a}^{+\pi / a} d k \sum_{n, n^{\prime}} \frac{\exp \left[-i k\left(n-n^{\prime}\right) a\right]}{D(k, s)+|Q(k, s)|} \times \\
\times\left[G_{0}(\mathbf{r}-n \mathbf{a}, E)+\bar{\chi} G_{0}(\mathbf{r}-n \mathbf{a}-\mathbf{g}, E)\right]\left[G_{0}\left(n \mathbf{a}-\mathbf{r}^{\prime}, E\right)+\chi G_{0}\left(n \mathbf{a}+\mathbf{g}-\mathbf{r}^{\prime}, E\right)\right] \\
-a \int_{-\pi / a}^{+\pi / a} d k \sum_{n, n^{\prime}} \frac{\exp \left[-i k\left(n-n^{\prime}\right) a\right]}{D(k, s)-|Q(k, s)|} \times \\
\times\left[G_{0}(\mathbf{r}-n \mathbf{a}, E)-\bar{\chi} G_{0}(\mathbf{r}-n \mathbf{a}-\mathbf{g}, E)\right]\left[G_{0}\left(n \mathbf{a}-\mathbf{r}^{\prime}, E\right)-\chi G_{0}\left(n \mathbf{a}+\mathbf{g}-\mathbf{r}^{\prime}, E\right)\right]
\end{gathered}
$$

where

$$
G_{0}(\mathbf{r}, E)=\frac{e^{i s r}}{4 \pi r}
$$

Expression (6) shows that two localized waveguide bands can appear near the chains. The dispersion law for one band is determined by roots of $D(k, s)+|Q(k, s)|$ of the second band - by $D(k, s)-|Q(k, s)|$. The corresponding wave functions have the forms:

$$
\begin{gathered}
\varphi_{ \pm}(k, \mathbf{r})= \\
{\left[\frac{d}{d E}(D(k, s) \pm|Q(k, s)|)\right]_{E=\varepsilon_{ \pm}(k)}^{-1 / 2}}
\end{gathered}
$$




$$
\sum_{n} \exp (-i k n a)\left[G_{0}(\mathbf{r}-n \mathbf{a}, E) \pm \bar{\chi} G_{0}(\mathbf{r}-n \mathbf{a}-\mathbf{g}, E)\right]_{E=\varepsilon_{ \pm}(k)}
$$

where $\varepsilon_{ \pm}(k)$ is determined from the equations:

$$
D\left(k, \sqrt{\frac{2 \mu E_{ \pm}}{h^{2}}}\right) \pm\left|Q\left(k, \sqrt{\frac{2 \mu E_{ \pm}}{h^{2}}}\right)\right|=0
$$

Consider the particular case for which the distance between the chains $g_{2}$ is greater than the distance between neighboring centers of one chain. Let us find the approximate expression for function $Q(k, s)$ if $s=i \chi, \chi$ is real. Using the formula:

$$
\frac{e^{-\chi \Delta}}{\Delta}=\frac{1}{\pi} \int_{-\infty}^{+\infty} \frac{e^{i p \Delta} d p}{p^{2}+\Delta^{2}}
$$

one transforms the expression for $Q(k, i \chi)$ to the following form:

$$
\begin{gathered}
Q(k, i \chi)=\frac{1}{\pi} \sum_{n=-\infty}^{+\infty} e^{i k n a} \int_{-\infty}^{+\infty} \frac{e^{i \xi \chi} d \xi}{\xi^{2}+\left(n a-g_{1}\right)^{2}+g_{2}^{2}}= \\
\frac{1}{\pi} \int_{-\infty}^{+\infty} e^{i \xi \chi}\left(\sum_{n=-\infty}^{+\infty} \frac{e^{i k n a}}{\xi^{2}+\left(n a-g_{1}\right)^{2}+g_{2}^{2}}\right) d \xi
\end{gathered}
$$

If $0 \leqslant k a<\pi$ then:

$$
\begin{gathered}
\sum_{n=-\infty}^{+\infty} \frac{e^{i k n a}}{\alpha^{2}+(n a-\beta)^{2}}= \\
-\frac{\pi}{2 a \alpha}\left\{\left[c t h\left[\frac{\pi}{a}(\beta-i \alpha)\right]-i\right] \exp [i k(\beta+i \alpha)]-\left[c t h\left[\frac{\pi}{a}(\beta-i \alpha)\right]-i\right] \exp [i k(\beta-i \alpha)]\right\}
\end{gathered}
$$

for $\alpha>0$. However, for $\alpha \rightarrow 0$ :

for $\alpha \rightarrow 0$.

$$
\begin{gathered}
\sum_{n=-\infty}^{+\infty} \frac{e^{i k n a}}{\alpha^{2}+(n a-\beta)^{2}} \simeq \\
\frac{\pi \exp (i k \beta)}{a \alpha}\left[\exp (-k \alpha)-\exp (k \alpha) \exp \left[-\frac{2 \pi}{a}(\alpha+\beta)\right]\right] .
\end{gathered}
$$

Hence,

$$
\begin{gathered}
Q(k, i \chi) \approx \frac{e^{i k g_{1}}}{a} \int_{-\infty}^{+\infty} \frac{e^{i \xi \chi}}{\sqrt{\xi^{2}+g_{2}^{2}}}\left[e^{-k \sqrt{\xi^{2}+g_{2}^{2}}}-e^{-2 \pi g_{1} / a} e^{(k-2 \pi / a) \sqrt{\xi^{2}+g_{1}^{2}}}\right] d \xi= \\
=\frac{e^{i k g_{1}}}{a} \int_{-\infty}^{+\infty} \frac{e^{i \eta \chi g_{2}}}{\sqrt{1+\eta^{2}}}\left[e^{-k g_{2} \sqrt{1+\eta^{2}}}-e^{-2 \pi g_{1} / a} e^{(k-2 \pi / a) g_{2} \sqrt{1+\eta^{2}}}\right] d \eta
\end{gathered}
$$

The last integral in (8) can be transformed to the integral over the curve which comprises the half-axis $\left(i, i_{\infty}\right)$ in upper half-plane. Thus,

$$
Q(k, i \chi) \approx \frac{2 e^{i k g_{1}}}{a} \int_{1}^{\infty} \frac{e^{-\theta \chi g_{2}}}{\sqrt{\theta^{2}-1}}\left[\cos \left(k g_{2}\right) \sqrt{\theta^{2}-1}-e^{-2 \pi g_{1} / a} \cos \left(\left(\frac{2 \pi}{a}-k\right) g_{2} \sqrt{\theta^{2}-1}\right) \approx\right]
$$




$$
\approx \sqrt{\frac{2 \pi}{g_{2}}} \frac{e^{i k g_{1}-k g_{2}}}{a}\left\{\frac{\sqrt{\chi+\sqrt{\chi^{2}+2 k^{2}}}}{\sqrt{\chi^{2}+2 k^{2}}}-e^{i 2 \pi g_{1} / a} \frac{\sqrt{\chi+\sqrt{\chi^{2}+2(2 \pi / a-k)^{2}}}}{\sqrt{\chi^{2}+2(2 \pi / a-k)^{2}}}\right\}
$$

Substituting (9) into (7) one determines the value of the waveguide band splitting caused by the existence of the second chain. Taking into account the smallness of $|Q(, i \chi)|$ for sufficiently remote chains, one finds that the level $\varepsilon_{0}(k)$ of single chain is split in the following manner:

$$
\varepsilon_{ \pm}(k)=\varepsilon_{0}(k) \pm \frac{h d \sqrt{2\left|\varepsilon_{0}(k)\right|}}{\sqrt{\mu} \sqrt{(d+\cos (k a))^{2}-1}}\left|Q\left(k, \frac{i}{h} \sqrt{2 \mu\left|\varepsilon_{0}(k)\right|}\right)\right|
$$

A gap in the waveguide band can appear if the top of the band $\varepsilon_{-}(k)$, i.e. $\varepsilon_{-}(\pi / a)$ is greater than the bottom $\varepsilon_{+}(0)$ of the band $\varepsilon_{+}(k)$, i.e. if $\varepsilon_{-}(\pi / a)<\varepsilon_{+}(0)$.

\section{The case of many chains}

We now consider the system of $M-1(M=2,3, \ldots)$ parallel identical chains. Let the position of centers be given by vectors $n \mathbf{a}_{\mathbf{1}}+m \mathbf{a}_{\mathbf{2}}, n, m-$ integer. In the framework of the zero-range potential approach, the wave function of the electron is $\Psi(\mathbf{r})$ :

$$
\lim _{p_{n m} \rightarrow 0}\left[\frac{\partial}{\partial p_{n m}}-b\right] p_{n m} \Psi=0
$$

where $p_{n m}=\left|\mathbf{r}-n \mathbf{a}_{\mathbf{1}}-m \mathbf{a}_{\mathbf{2}}\right|$. We will seek the perturbed Green function $G\left(\mathbf{r}, \mathbf{r}^{\prime}, E\right)$ for $\Im E>0$ in the following form:

$$
G\left(\mathbf{r}, \mathbf{r}^{\prime}, E\right)=G_{0}\left(\mathbf{r}-\mathbf{r}^{\prime}, E\right)+4 \pi \sum_{n, m} C_{n m} G_{0}\left(\mathbf{r}-n \mathbf{a}_{\mathbf{1}}-m \mathbf{a}_{\mathbf{2}}, E\right),
$$

where $G_{0}(\mathbf{r}, E)$ is the Green function for free space. Conditions (10) gives one a system for coefficients $C_{n m}$ :

$$
(i s-b) C_{n m}+\sum_{n^{\prime} \neq n, m^{\prime} \neq m} C_{n^{\prime} m^{\prime}} \frac{\exp \left[i s\left|\left(n-n^{\prime}\right) \mathbf{a}_{\mathbf{1}}+\left(m-m^{\prime}\right) \overrightarrow{a_{2}}\right|\right]}{\left|\left(n-n^{\prime}\right) \mathbf{a}_{\mathbf{1}}+\left(m-m^{\prime}\right) \mathbf{a}_{\mathbf{2}}\right|}=\varphi_{n m}\left(\mathbf{r}^{\prime}\right),
$$

where

$$
\varphi_{n m}\left(\mathbf{r}^{\prime}\right)=-\frac{1}{4 \pi} \frac{\exp \left[i s\left|\mathbf{r}^{\prime}-n \mathbf{a}_{\mathbf{1}}-m \mathbf{a}_{\mathbf{2}}\right|\right]}{\left|\mathbf{r}^{\prime}-n \mathbf{a}_{\mathbf{1}}-m \mathbf{a}_{\mathbf{2}}\right|} .
$$

The prime sign near the sum means that it is sufficient to satisfy only one from the conditions $n^{\prime} \neq n$ or $m \neq m^{\prime}$. , To simplify formulas for $C_{n m}$, we assume that $\left|C_{n, m \pm p}\right| \ll\left|C_{n, m \pm 1}\right|$ for $p>1$. Then, after the Fourier transform along the chain, one obtains:

$$
D(k, s) \xi_{m}(k)+D_{1}(k, s)\left[\xi_{m+1}(k)+\xi_{m-1}(k)\right]=\eta_{m}\left(k, \mathbf{r}^{\prime}\right),
$$

where

$$
\begin{gathered}
\xi_{m}(k)=\sum_{n} C_{n m} \exp (i k n a) \\
D(k, s)=\frac{1}{a} \ln \frac{d}{\cos \left(a_{1} s\right)-\cos \left(a_{2} k\right)}, \\
D_{1}(k, s)=\sum_{n=-\infty}^{+\infty} \frac{\exp \left[i s\left|n \mathbf{a}_{\mathbf{1}}+\mathbf{a}_{\mathbf{2}}\right|+i k n a_{1}\right]}{\left|n \mathbf{a}_{\mathbf{1}}+\mathbf{a}_{\mathbf{2}}\right|}
\end{gathered}
$$




$$
\eta_{m}\left(k, \mathbf{r}^{\prime}\right)=-\frac{1}{4 \pi} \sum_{n} \varphi_{n m}\left(\mathbf{r}^{\prime}\right) \exp (i k n a)
$$

The dispersion equation is obtained from the solvability condition for the following homogeneous difference equation:

$$
D \xi_{m}+D_{1}\left(\xi_{m+1}+\xi_{m-1}\right)=0 .
$$

The solution of (13) has the form:

$$
\xi_{m}(k)=\Xi_{1}(k) \cos \left(k_{2} m a_{2}\right)+\Xi_{2}(k) \sin \left(k_{2} m a_{2}\right) .
$$

The range for $k_{2}$ and value of $\Xi_{1}$ (for $M-1$ chains) are determined from the boundary conditions $C_{n 0}=C_{n M}=0$. Consequently, $\xi_{0}(k)=\xi_{M}(k)=0, \Xi_{1}(k)=0$ and

$$
k_{2}=\pi l /\left(M a_{2}\right) ; \quad l=1,2, \ldots, M-1 .
$$

If one inserts the solution:

$$
\xi_{m}(k)=\Xi(k) \sin (\pi m l / M),
$$

into (11), the dispersion equation is obtained:

$$
D(k, s)+2 D_{1}(k, s) \cos (\pi l / M)=0 .
$$

Waveguide bands are given by the solution $s=i \chi, \chi>0$, of (14). Taking into account (12), one reduces (14) to the following form:

$$
\cosh \left(a_{1} \chi\right)=\cos \left(a_{1} k\right)+d_{1}(\chi, l),
$$

where $d_{1}(\chi, l)=d \exp \left[2 a_{1} D_{1} \cos (\pi l) / M\right]$, or, correspondingly, to the form:

$$
E_{1}(k)=-\frac{h^{2}}{2 \mu a_{1}}\left\{\ln \left[d_{1}(\chi, l)+\cos \left(a_{1} k\right)+\sqrt{\left[d_{1}(\chi, l)+\cos \left(a_{1} k\right)\right]^{2}-1}\right]\right\}^{2},
$$

where $E_{1}(k)=-\frac{h^{2} \chi^{2}}{2 \mu}$. Note that for small $k, \chi$, one can replace the sum for $D_{1}$ from $(12)$ by the integral:

$$
D_{1} \approx \int_{-\infty}^{+\infty} \frac{\exp \left[-\chi\left|\nu \mathbf{a}_{\mathbf{1}}+\mathbf{a}_{\mathbf{2}}\right|+i k \nu a_{1}\right]}{\left|\nu \mathbf{a}_{\mathbf{1}}+\mathbf{a}_{\mathbf{2}}\right|} d \nu=\frac{2}{a_{1}} K_{0}\left(\sqrt{\left(\chi a_{1}\right)^{2}+\left(k a_{2}\right)^{2}}\right)
$$

where $K_{0}(z)$ is the Macdonald function of zero order. Due to the integer parameter $l$, equation (15) determines, generally speaking, $M-1$ waveguide bands. The conductivity is determined by such bands which are empty or partially filled, i.e. contain the Fermi level $E_{F}$. If each atom of the chain has one "free" electron, then the Fermi level $E_{F}$ is determined from the equation:

$$
\frac{\pi(M-1)}{2 a_{1}}=\sum_{l=1}^{M-1} \int_{E_{0}}^{E_{F}} \frac{d k}{d E} d E .
$$

Taking into account (15), one rewrites (16) in the form:

$$
\frac{\pi}{2 a_{1}}=\frac{1}{M-1} \sum_{l=1}^{M-1} \int_{\chi_{0}}^{\chi_{F}} \frac{s h\left(\chi a_{1}\right)-d_{1}^{\prime}(\chi, l)}{\sqrt{1-\left[\operatorname{ch}\left(\chi a_{1}\right)-d_{1}\right]^{2}}} d \chi,
$$


where $d_{1}^{\prime}(\chi, l)=\frac{\partial d_{1}(\chi, l)}{\partial\left(\chi a_{1}\right)}$. If one ignores the difference between $d_{1}(\chi, l)$ and $d$, then equation (17) gives one the following value of the Fermi level:

$$
E_{F}^{0}=-\frac{h^{2}}{2 \mu a_{1}^{2}} \ln ^{2}\left[d+\sqrt{d^{2}-1}\right]
$$

which corresponds to the case of single chain.

\section{Acknowledgments}

This work was partially financially supported by the Government of the Russian Federation (grant 074-U01), by Ministry of Science and Education of the Russian Federation (GOSZADANIE 2014/190, Projects No 14.Z50.31.0031 and No. 1.754.2014/K), by grant MK-5001.2015.1 of the President of the Russian Federation, and by grant of St. Petersburg.

\section{References}

[1] I.Yu. Popov. On the possibility of magnetoresistance governed by light. Nanosystems: Phys. Chem. Math., 2013, 4(6), P. 795-799.

[2] U. Wulf, M. Krahlisch, J. Kucera, H. Richter, J. Hontschel. A quantitative model for quantum transport in nano-transistors. Nanosystems: Phys. Chem. Math., 2013, 4(6), P. 800-809.

[3] I. S. Lobanov, A. I. Trifanov, E. S. Trifanova. Genetic algorithm for constructing graphene nanoribbon with given electronic transport properties. Nanosystems: Phys. Chem. Math., 2013, 4(4), P. 512-523.

[4] I.Yu.Popov, A.N.Skorynina, I.V.Blinova. On the existence of point spectrum for branching strips quantum graph. J. Math. Phys., 2014, 55, 033504/1-20.

[5] A.A.Boitsev, I.Yu.Popov, O.V.Sokolov. Hamiltonian with zero-range potentials having infinite number of eigenvalues. Nanosystems: Phys. Chem. Math., 2012, 3(4), P. 9-19.

[6] S.Albeverio, F.Gesztesy, R.Hoegh-Krohn, H.Holden with an appendix by P.Exner 2005 Solvable Models in Quantum Mechanics: Second Edition. (Providence, R.I.: AMS Chelsea Publishing)

[7] B.S.Pavlov, Extensions theory and explicitly solvable models. Uspekhi. Mat. Nauk., 1987, 42(6), P. 99131.

[8] J.Berndt, M. M. Malamud, H. Neidhardt. Scattering matrices and Weyl functions. Proc. London Math. Soc., 2008, 97(3), P. 568-598.

[9] A.A. Boitsev, H. Neidhardt, I.Yu. Popov. Weyl function for sum of operators tensor products. Nanosystems: Phys. Chem. Math., 2013, 4(6), P. 747-759.

[10] M. S. Birman, M. Z. Solomyak. Spectral Theory of Self-Adjoint Operators in Hilbert Space (D. Reidel Publishing Company, Dordrecht-Boston-Lancaster, 1987).

[11] A. Alonso, B. Simon. The Birman-Krein-Vishik theory of selfadjoint extensions of semi-bounded operators. J. Oper. Theory, 1980, 4, P. 251-270.

[12] V.M. Adamyan, B.S. Pavlov. Null-range potentials and M.G. Krein's formula for generalized resolvents. Journal of Soviet Mathematics, 1988, 42(2), P. 1537-1550. 